\title{
On some classes of difference equations of infinite order
}

\author{
Alexander V Vasilyev ${ }^{1}$ and Vladimir B Vasilyev2 ${ }^{2 *}$
}

${ }^{\text {*Correspondence: }}$

vladimir.b.vasilyev@gmail.com

${ }^{2}$ Chair of Pure Mathematics, Lipetsk

State Technical University,

Moskovskaya 30, Lipetsk, 398600,

Russia

Full list of author information is

available at the end of the article

\begin{abstract}
We consider a certain class of difference equations on an axis and a half-axis, and we establish a correspondence between such equations and simpler kinds of operator equations. The last operator equations can be solved by a special method like the Wiener-Hopf method.
\end{abstract}

MSC: $39 \mathrm{~B} 32 ; 42 \mathrm{~A} 38$

Keywords: difference equation; symbol; solvability

\section{Introduction}

Difference equations of finite order arise very often in various problems in mathematics and applied sciences, for example in mathematical physics and biology. The theory for solving such equations is very full for equations with constant coefficients [1, 2], but fully incomplete for the case of variable coefficients. Some kinds of such equations were obtained by the second author by studying general boundary value problems for mode elliptic pseudo differential equations in canonical non-smooth domains, but there is no solution algorithm for all situations [3-5]. There is a certain intermediate case between the two mentioned above, namely it is a difference equation with constant coefficients of infinite order. Here we will briefly describe these situations.

The general form of the linear difference equation of order $n$ is the following [1,2]:

$$
\sum_{k=0}^{n} a_{k}(x) u(x+k)=v(x), \quad x \in M \subset \mathbb{R}
$$

where the functions $a_{k}(x), k=1, \ldots, n, v(x)$ are defined on $M$ and given, and $u(x)$ is an unknown function. Since $n \in \mathbf{N}$ is an arbitrary number and all points $x, x+1, \ldots, x+n$, $\forall x \in M$, should be in the set $M$, this set $M$ may be a ray from a certain point or the whole $\mathbf{R}$.

A more general type of difference equation of finite order is the equation

$$
\sum_{k=0}^{n} a_{k}(x) u\left(x+\beta_{k}\right)=v(x), \quad x \in M \subset \mathbb{R},
$$

where $\left\{\beta_{k}\right\}_{k=0}^{n} \subset \mathbb{R}$.

Further, such equations can be equations with a continuous variable or a discrete one, and this property separates such an equation on a class of properly difference equations

(c) 2015 Vasilyev and Vasilyev. This article is distributed under the terms of the Creative Commons Attribution 4.0 International License (http://creativecommons.org/licenses/by/4.0/), which permits unrestricted use, distribution, and reproduction in any medium, provided you give appropriate credit to the original author(s) and the source, provide a link to the Creative Commons license, and indicate if changes were made. 
and discrete equations. In this paper we will consider the case of a continuous variable $x$, and a solution on the right-hand side will be considered in the space $L_{2}(\mathbb{R})$ for all equations.

\subsection{Difference equation of a finite order with constant coefficients}

This is an equation of the type

$$
\sum_{k=0}^{n} a_{k} u\left(x+\beta_{k}\right)=v(x), \quad x \in \mathbb{R}
$$

and it easily can be solved by the Fourier transform:

$$
(F u)(\xi) \equiv \tilde{u}(\xi)=\int_{-\infty}^{+\infty} e^{-i x \cdot \xi} u(x) d x
$$

Indeed, applying the Fourier transform to (3) we obtain

$$
\tilde{u}(\xi) \sum_{k=0}^{n} a_{k} e^{i \beta_{k} \xi}=\tilde{v}(\xi)
$$

or renaming

$$
\tilde{u}(\xi) p_{n}(\xi)=\tilde{v}(\xi)
$$

The function $p_{n}(\xi)$ is called a symbol of a difference operator on the left-hand side (3) (cf. [6]). If $p_{n}(\xi) \neq 0, \forall \xi \in \mathbb{R}$, then (3) can easily be solved,

$$
u(x)=F_{\xi \rightarrow x}^{-1}\left(p_{n}^{-1}(\xi) \tilde{v}(\xi)\right)
$$

\subsection{Difference equation of infinite order with constant coefficients}

The same arguments are applicable for the case of an unbounded sequence $\left\{\beta_{k}\right\}_{-\infty}^{+\infty}$. Then the difference operator with complex coefficients

$$
\mathcal{D}: u(x) \longmapsto \sum_{-\infty}^{+\infty} a_{k} u\left(x+\beta_{k}\right), \quad x \in \mathbb{R}, a_{k} \in \mathbb{C}
$$

has the following symbol:

$$
\sigma(\xi)=\sum_{-\infty}^{+\infty} a_{k} e^{i \beta_{k} \xi}
$$

Lemma 1 The operator $\mathcal{D}$ is a linear bounded operator $L_{2}(\mathbb{R}) \rightarrow L_{2}(\mathbb{R})$ if $\left\{a_{k}\right\}_{-\infty}^{+\infty} \in l^{1}$.

Proof The proof of this assertion can be obtained immediately.

If we consider the operator (4) for $x \in \mathbb{Z}$ only

$$
\mathcal{D}: u\left(x_{d}\right) \longmapsto \sum_{-\infty}^{+\infty} a_{k} u\left(x_{d}+\beta_{k}\right), \quad x_{d} \in \mathbb{Z},
$$


then its symbol can be defined by the discrete Fourier transform $[7,8]$

$$
\sigma_{d}(\xi)=\sum_{-\infty}^{+\infty} a_{k} e^{i \beta_{k} \xi}, \quad \xi \in[-\pi, \pi] .
$$

\subsection{Difference and discrete equations}

Obviously there are some relations between difference and discrete equations. Particularly, if $\left\{\beta_{k}\right\}_{-\infty}^{+\infty}=\mathbb{Z}$, then the operator (5) is a discrete convolution operator. For studying discrete operators in a half-space the authors have developed a certain analytic technique [9-11]. Below we will try to enlarge this technique for more general situations.

\section{General difference equations}

We consider the equation

$$
(\mathcal{D} u)(x)=v(x), \quad x \in \mathbb{R}_{+},
$$

where $\mathbb{R}_{+}=\{x \in \mathbb{R}, x>0\}$.

For studying this equation we will use methods of the theory of multi-dimensional singular integral and pseudo differential equations $[3,6,12]$ which are non-usual in the theory of difference equations. Our next goal is to study multi-dimensional difference equations, and this one-dimensional variant is a model for considering other complicated situations. This approach is based on the classical Riemann boundary value problem and the theory of one-dimensional singular integral equations [13-15].

\subsection{Background}

The first step is the following. We will use the theory of so-called paired equations [15] of the type

$$
\left(a P_{+}+b P_{-}\right) U=V
$$

in the space $L_{2}(\mathbb{R})$, where $a, b$ are convolution operators with corresponding functions $a(x), b(x), x \in \mathbb{R}, P_{ \pm}$are projectors on the half-axis $\mathbb{R}_{ \pm}$. More precisely,

$$
\left(a P_{+} U\right)(x)=\int_{0}^{+\infty} a(x-y) U(y) d y, \quad\left(b P_{-} U\right)(x)=\int_{-\infty}^{0} b(x-y) U(y) d y .
$$

Applying the Fourier transform to (7) we obtain [12] the following one-dimensional singular integral equation [13-15]:

$$
\tilde{a}(\xi)(P \tilde{U})(\xi)+\tilde{b}(\xi)(Q \tilde{U})(\xi)=\tilde{V}(\xi)
$$

where $P, Q$ are two projectors related to the Hilbert transform

$$
\begin{aligned}
& (H u)(x)=\text { v.p. } \frac{1}{\pi i} \int_{-\infty}^{+\infty} \frac{u(y)}{x-y} d y, \\
& P=\frac{1}{2}(I+H), \quad Q=\frac{1}{2}(I-H) .
\end{aligned}
$$


Equation (8) is closely related to the Riemann boundary value problem $[13,14]$ for upper and lower half-planes. We now recall the statement of the problem: finding a pair of functions $\Phi^{ \pm}(\xi)$ which admit an analytic continuation on upper $\left(\mathbb{C}_{+}\right)$and lower $\left(\mathbb{C}_{-}\right)$ half-planes in the complex plane $\mathbb{C}$ and of which their boundary values on $\mathbb{R}$ satisfy the following linear relation:

$$
\Phi^{+}(\xi)=G(\xi) \Phi^{-}(\xi)+g(\xi)
$$

where $G(\xi), g(\xi)$ are given functions on $\mathbb{R}$.

There is a one-to-one correspondence between the Riemann boundary value problem (9) and the singular integral equation (8), and

$$
G(\xi)=\tilde{a}^{-1}(\xi) \tilde{b}(\xi), \quad \tilde{V}(\xi)=\tilde{a}^{-1}(\xi) g(\xi) .
$$

\subsection{Topological barrier}

We suppose that the symbol $G(\xi)$ is a continuous non-vanishing function on the compactification $\dot{\mathbb{R}}(G(\xi) \neq 0, \forall \xi \in \dot{\mathbb{R}})$ and

$$
\text { Ind } G \equiv \frac{1}{2 \pi} \int_{-\infty}^{+\infty} d \arg G(t)=0
$$

The last condition (10), is necessary and sufficient for the unique solvability of the problem $(9)$ in the space $L_{2}(\mathbb{R})[13,14]$. Moreover, the unique solution of the problem (9) can be constructed with a help of the Cauchy type integral

$$
\Phi^{+}(t)=G_{+}(t) P\left(G_{+}^{-1}(t) g(t)\right), \quad \Phi^{-}(t)=-G_{-}^{-1}(t) Q\left(G_{+}^{-1}(t) g(t)\right),
$$

where $G_{ \pm}$are factors of a factorization for the $G(t)$ (see below),

$$
G_{+}(t)=\exp (P(\ln G(t))), \quad G_{-}(t)=\exp (Q(\ln G(t)))
$$

\subsection{Difference equations on a half-axis}

Equation (6) can easily be transformed into (7) in the following way. Since the right-hand side in (6) is defined on $\mathbb{R}_{+}$only we will continue $v(x)$ on the whole $\mathbb{R}$ so that this continuation lf $\in L_{2}(\mathbb{R})$. Further we will rename the unknown function $u_{+}(x)$ and define the function

$$
u_{-}(x)=(l f)(x)-\left(\mathcal{D} u_{+}\right)(x)
$$

Thus, we have the following equation:

$$
\left(\mathcal{D} u_{+}\right)(x)+u_{-}(x)=(l f)(x), \quad x \in \mathbb{R}
$$

which holds for the whole space $\mathbb{R}$.

After the Fourier transform we have

$$
\sigma(\xi) \tilde{u}_{+}(\xi)+\tilde{u}_{-}(\xi)=\tilde{l v}(\xi)
$$

where $\sigma(\xi)$ is called a symbol of the operator $\mathcal{D}$. 
To describe a solving technique for (11) we recall the following $(c f .[13,14])$.

Definition A factorization for an elliptic symbol is called its representation if it is the form

$$
\sigma(\xi)=\sigma_{+}(\xi) \cdot \sigma_{-}(\xi)
$$

where the factors $\sigma_{+}, \sigma_{-}$admit an analytic continuation into the upper and lower complex half-planes $\mathbb{C}_{ \pm}$, and $\sigma_{ \pm}^{ \pm 1} \in L_{\infty}(\mathbb{R})$.

Example 1 Let us consider the Cauchy type integral

$$
\frac{1}{2 \pi i} \int_{-\infty}^{+\infty} \frac{u(t)}{z-t} d t \equiv \Phi(z), \quad z=x \pm i y .
$$

It is well known this construction plays a crucial role for a decomposition $L_{2}(\mathbb{R})$ on two orthogonal subspaces, namely

$$
L_{2}(\mathbb{R})=A_{+}(\mathbb{R}) \oplus A_{-}(\mathbb{R}),
$$

where $A_{ \pm}(\mathbb{R})$ consists of functions admitting an analytic continuation onto $\mathbb{C}_{ \pm}$.

The boundary values of the integral $\Phi(z)$ satisfy the Plemelj-Sokhotskii formulas [13, 14], and thus the projectors $P$ and $Q$ are corresponding projectors on the spaces of analytic functions [15].

The simple example we need is

$$
\exp (u)=\exp (P u) \cdot \exp (Q u)
$$

Theorem 2 Let $\sigma(\xi) \in C(\dot{\mathbb{R}})$, Ind $\sigma=0$. Then (6) has unique solution in the space $L_{2}\left(\mathbb{R}_{+}\right)$ for arbitrary right-hand side $v \in L_{2}\left(\mathbb{R}_{+}\right)$, and its Fourier transform is given by the formula

$$
\tilde{u}(\xi)=\frac{1}{2} \sigma^{-1}(\xi) \widetilde{l v}(\xi)+\frac{\sigma_{+}^{-1}(\xi)}{2 \pi i} \text { v.p. } \int_{-\infty}^{+\infty} \frac{\sigma_{-}^{-1}(\eta) \widetilde{l v}(\eta)}{\xi-\eta} d \eta .
$$

Proof We have

$$
\sigma_{+}(\xi) \tilde{u}_{+}(\xi)+\sigma_{-}^{-1}(\xi) \tilde{u}_{-}(\xi)=\sigma_{-}^{-1}(\xi) \tilde{l} v(\xi) .
$$

Further, since $\sigma_{-}^{-1}(\xi) \widetilde{l v}(\xi) \in L_{2}(\mathbb{R})$ we decompose it into two summands

$$
\sigma_{-}^{-1}(\xi) \tilde{l v}(\xi)=P\left(\sigma_{-}^{-1}(\xi) \tilde{l}(\xi)\right)+Q\left(\sigma_{-}^{-1}(\xi) \tilde{l v}(\xi)\right)
$$

and write

$$
\sigma_{+}(\xi) \tilde{u}_{+}(\xi)-P\left(\sigma_{-}^{-1}(\xi) \tilde{l v}(\xi)\right)=Q\left(\sigma_{-}^{-1}(\xi) \tilde{l v}(\xi)\right)-\sigma_{-}^{-1}(\xi) \tilde{u}_{-}(\xi) .
$$

The left-hand side of the last quality belongs to the space $A_{+}(\mathbb{R})$, but the right-hand side belongs to $A_{-}(\mathbb{R})$, consequently these are zeros. Thus,

$$
\sigma_{+}(\xi) \tilde{u}_{+}(\xi)-P\left(\sigma_{-}^{-1}(\xi) \tilde{l}(\xi)\right)=0
$$


and

$$
\tilde{u}_{+}(\xi)=\sigma_{+}^{-1}(\xi) P\left(\sigma_{-}^{-1}(\xi) \widetilde{l v}(\xi)\right)
$$

or in the complete form

$$
\tilde{u}_{+}(\xi)=\frac{1}{2} \sigma^{-1}(\xi) \tilde{l} v(\xi)+\frac{\sigma_{+}^{-1}(\xi)}{2 \pi i} \mathrm{v} \cdot \mathrm{p} \cdot \int_{-\infty}^{+\infty} \frac{\sigma_{-}^{-1}(\eta) \tilde{l v}(\eta)}{\xi-\eta} d \eta
$$

Remark 1 This result does not depend on the continuation $l v$. Let us denote by $M_{ \pm}(x)$ the inverse Fourier images of the functions $\sigma_{ \pm}^{-1}(\xi)$. Indeed, (12) leads to the following construction:

$$
\begin{aligned}
u_{+}(x) & =\int_{-\infty}^{+\infty} M_{+}(x-y)\left(\int_{0}^{+\infty} M_{-}(y-t)(l v)(t) d t\right) d y \\
& =\int_{-\infty}^{+\infty} M_{+}(x-y)\left(\int_{0}^{+\infty} M_{-}(y-t) v(t) d t\right) d y
\end{aligned}
$$

Remark 2 The condition $\sigma(\xi) \in C(\dot{\mathbb{R}})$ is not a strong restriction. Such symbols exist for example in the case that $\sigma(\xi)$ is represented by a finite sum, and $\beta_{k} \in \mathbb{Q}$. Then $\sigma(\xi)$ is a continuous periodic function.

\section{General solution}

Since $\sigma(\xi) \in C(\dot{\mathbb{R}})$, and Ind $\sigma$ is an integer, we consider the case $æ \equiv$ Ind $\sigma \in \mathbb{N}$ in this section.

Theorem 3 Let Ind $\sigma \in \mathbb{N}$. Then a general solution of (6) in the Fourier image can be written in the form

$$
\tilde{u}_{+}(\xi)=\sigma_{+}^{-1}(\xi) \omega^{-\mathfrak{x}}(\xi) P\left(\sigma_{-}^{-1}(\xi) \tilde{l}(\xi)\right)+(\xi-i)^{-\mathfrak{x}} \sigma_{+}^{-1}(\xi) P_{\mathfrak{x}-1}(\xi),
$$

and it depends on $\mathfrak{x}$ arbitrary constants.

Proof The function

$$
\omega(\xi)=\frac{\xi-i}{\xi+i}
$$

has the index 1 [13-15], thus the function

$$
\omega^{-\mathfrak{x}}(\xi) \sigma(\xi)=\left(\frac{\xi-i}{\xi+i}\right)^{-\mathfrak{x}} \cdot \sigma(\xi)
$$

has the index 0 , and we can factorize this function

$$
\omega^{-\mathfrak{x}}(\xi) \sigma(\xi)=\sigma_{+}(\xi) \sigma_{-}(\xi) .
$$

Further, we write after (11)

$$
\omega^{\mathfrak{x}}(\xi) \omega^{-\mathfrak{x}}(\xi) \sigma(\xi) \tilde{u}_{+}(\xi)+\tilde{u}_{-}(\xi)=\tilde{l} \nu(\xi),
$$


factorize $\omega^{-\mathfrak{x}}(\xi) \sigma(\xi)$, and rewrite

$$
\omega^{\mathfrak{x}}(\xi) \sigma_{+}(\xi) \tilde{u}_{+}(\xi)+\sigma_{-}^{-1}(\xi) \tilde{u}_{-}(\xi)=\sigma_{-}^{-1}(\xi) \tilde{l v}(\xi)
$$

Taking into account our notations we have

$$
\begin{aligned}
& \sigma_{+}(\xi) \tilde{u}_{+}(\xi)+\omega^{-\mathfrak{x}}(\xi) \sigma_{-}^{-1}(\xi) \tilde{u}_{-}(\xi) \\
& \quad=\omega^{-\mathfrak{x}}(\xi) P\left(\sigma_{-}^{-1}(\xi) \tilde{l v}(\xi)\right)+\omega^{-\mathfrak{x}}(\xi) Q\left(\sigma_{-}^{-1}(\xi) \tilde{l v}(\xi)\right),
\end{aligned}
$$

because

$$
\sigma_{-}^{-1}(\xi) \widetilde{l v}(\xi)=P\left(\sigma_{-}^{-1}(\xi) \widetilde{l v}(\xi)\right)+Q\left(\sigma_{-}^{-1}(\xi) \widetilde{l v}(\xi)\right)
$$

and

$$
\begin{aligned}
& \sigma_{+}(\xi) \tilde{u}_{+}(\xi)-\omega^{-\mathfrak{x}}(\xi) P\left(\sigma_{-}^{-1}(\xi) \tilde{l v}(\xi)\right) \\
& \quad=\omega^{-\mathfrak{x}}(\xi) Q\left(\sigma_{-}^{-1}(\xi) \tilde{l v}(\xi)\right)-\omega^{-\mathfrak{x}}(\xi) \sigma_{-}^{-1}(\xi) \tilde{u}_{-}(\xi)
\end{aligned}
$$

and we conclude from the last that the left-hand side and the right-hand side also are a polynomial $P_{\mathfrak{x}-1}(\xi)$ of order $\mathfrak{x}-1$. It follows from the generalized Liouville theorem [13, 14 ] because the left-hand side has one pole of order $æ$ in $\mathbb{C}$ in the point $z=i$. So, we have

$$
\tilde{u}_{+}(\xi)=\sigma_{+}^{-1}(\xi) \omega^{-\mathfrak{x}}(\xi) P\left(\sigma_{-}^{-1}(\xi) \widetilde{l}(\xi)\right)+(\xi-i)^{-\mathfrak{x}} \sigma_{+}^{-1}(\xi) P_{\mathfrak{x}-1}(\xi) .
$$

Remark 3 This result does not depend on the choice of the continuation $l$.

Corollary 4 Let $v(x) \equiv 0, æ \in \mathbb{N}$. Then a general solution of the homogeneous equation (6) is given by the formula

$$
\tilde{u}_{+}(\xi)=(\xi-i)^{-\mathfrak{x}} \sigma_{+}^{-1}(\xi) P_{\mathfrak{x}-1}(\xi) .
$$

\section{Solvability conditions}

Theorem 5 Let -Ind $\sigma \in \mathbb{N}$. Then (6) has a solution from $L_{2}\left(\mathbb{R}_{+}\right)$iff the following conditions hold:

$$
\int_{-\infty}^{+\infty}(\xi-i)^{k-1} \sigma_{-}^{-1}(\xi) \tilde{l \nu}(\xi) d \xi=0, \quad k=1,2, \ldots,|æ| .
$$

Proof We argue as above and use the equality (14); we write it as

$$
\begin{aligned}
& (\xi-i)^{\mathfrak{x}} \sigma_{+}(\xi) \tilde{u}_{+}(\xi)-(\xi+i)^{\mathfrak{x}} P\left(\sigma_{-}^{-1}(\xi) \widetilde{l v}(\xi)\right) \\
& \quad=(\xi+i)^{\mathfrak{x}} Q\left(\sigma_{-}^{-1}(\xi) \widetilde{l v}(\xi)\right)-(\xi+i)^{\mathfrak{x}} \sigma_{-}^{-1}(\xi) \tilde{u}_{-}(\xi) .
\end{aligned}
$$

Since we work with $L_{2}(\mathbb{R})$ both the left-hand side and the right-hand side are equal to zero at infinity, hence these are zeros, and

$$
\tilde{u}_{+}(\xi)=\sigma_{+}^{-1}(\xi)\left(\frac{\xi-i}{\xi+i}\right)^{-\mathfrak{x}} P\left(\sigma_{-}^{-1}(\xi) \tilde{l v}(\xi)\right)
$$


But there is some inaccuracy. Indeed, this solution belongs to the space $A_{+}(\mathbb{R})$, but more exactly it belongs to its subspace $A_{+}^{k}(\mathbb{R})$. This subspace consists of functions analytic in $\mathbb{C}_{+}$ with zeros of the order $-\mathfrak{x}$ in the point $z=i$. To obtain a solution from $L_{2}\left(\mathbb{R}_{+}\right)$we need some corrections in the last formula. Since the operator $P$ is related to the Cauchy type integral we will use certain decomposition formulas for this integral (see also [12-14]).

Let us denote $\sigma_{-}^{-1}(\xi) \widetilde{l v}(\xi) \equiv g(\xi)$ and consider the following integral:

$$
\int_{-\infty}^{+\infty} \frac{g(\eta) d \eta}{z-\eta}, \quad z=\xi+i \tau \in \mathbb{C}
$$

Using a simple formula for a kernel

$$
\frac{1}{z-\eta}=\sum_{k=1}^{\mid \mathfrak{x}} \frac{(\eta-i)^{k-1}}{(z-i)^{k}}+\frac{(\eta-i)^{|\mathfrak{}|}}{(z-i)^{|\mathfrak{x}|}(z-\eta)}
$$

we obtain the following decomposition:

$$
\int_{-\infty}^{+\infty} \frac{g(\eta) d \eta}{z-\eta}=\sum_{k=1}^{|\mathfrak{x}|}(z-i)^{-k} \int_{-\infty}^{+\infty}(\eta-i)^{k-1} g(\eta) d \eta+\int_{-\infty}^{+\infty} \frac{(\eta-i)^{|\mathfrak{x}|} g(\eta) d \eta}{(z-i)^{|\mathfrak{x}|}(z-\eta)}
$$

So, we have a following property. If the conditions

$$
\int_{-\infty}^{+\infty}(\eta-i)^{k-1} g(\eta) d \eta=0, \quad k=1,2, \ldots,|æ|
$$

hold, then we obtain

$$
\int_{-\infty}^{+\infty} \frac{g(\eta) d \eta}{z-\eta}=(z-i)^{\mathfrak{x}} \int_{-\infty}^{+\infty} \frac{(\eta-i)^{|\mathfrak{x}|} g(\eta) d \eta}{z-\eta}
$$

Hence the boundary values on $\mathbb{R}$ for the left-hand side and the right-hand one are equal, and thus

$$
P(g(\xi))=(\xi-i)^{\mathfrak{x}} P\left((\xi-i)^{|\mathfrak{x}|} g(\xi)\right)
$$

Substituting the last formula into the solution formula we write

$$
\tilde{u}_{+}(\xi)=\sigma_{+}^{-1}(\xi)(\xi+i)^{\mathfrak{x}} P\left((\xi-i)^{|\mathfrak{x}|} \sigma_{-}^{-1}(\xi) \widetilde{l v}(\xi)\right)
$$

\section{Conclusion}

It seems this approach to difference equations may be useful for studying the case that the variable $x$ is a discrete one. We have some experience in the theory of discrete equations [9-11], and we hope that we can be successful in this situation also. Moreover, in our opinion the developed methods might be applicable for multi-dimensional difference equations. 
Authors' contributions

All authors contributed equally to the writing of this paper. All authors read and approved the final manuscript.

\section{Author details}

'Department of Mathematical Analysis, National Research Belgorod State University, Studencheskaya 14/1, Belgorod, 307008, Russia. ${ }^{2}$ Chair of Pure Mathematics, Lipetsk State Technical University, Moskovskaya 30, Lipetsk, 398600, Russia.

\section{Acknowledgements}

The authors are very grateful to the anonymous referees for their valuable suggestions. This work is supported by Russian Fund of Basic Research and government of Lipetsk region of Russia, project No. 14-41-03595-a.

Received: 4 March 2015 Accepted: 17 June 2015 Published online: 10 July 2015

\section{References}

1. Milne-Thomson, LM: The Calculus of Finite Differences. Chelsea, New York (1981)

2. Jordan, C: Calculus of Finite Differences. Chelsea, New York (1950)

3. Vasil'ev, VB: Wave Factorization of Elliptic Symbols: Theory and Applications. Introduction to the Theory of Boundary Value Problems in Non-Smooth Domains. Kluwer Academic, Dordrecht (2000)

4. Vasilyev, VB: General boundary value problems for pseudo differential equations and related difference equations. Adv. Differ. Equ. 2013, 289 (2013)

5. Vasilyev, VB: On some difference equations of first order. Tatra Mt. Math. Publ. 54, 165-181 (2013)

6. Mikhlin, SG, Prößdorf, S: Singular Integral Operators. Akademie Verlag, Berlin (1986)

7. Sobolev, SL: Cubature Formulas and Modern Analysis: An Introduction. Gordon \& Breach, Montreux (1992)

8. Dudgeon, DE, Mersereau, RM: Multidimensional Digital Signal Processing. Prentice Hall, Englewood Cliffs (1984)

9. Vasilyev, AV, Vasilyev, VB: Discrete singular operators and equations in a half-space. Azerb. J. Math. 3(1), 84-93 (2013)

10. Vasilyev, AV, Vasilyev, VB: Discrete singular integrals in a half-space. In: Current Trends in Analysis and Its Applications, Proc. 9th Congress, Krakow, Poland, August 2013, pp. 663-670. Birkhäuser, Basel (2015)

11. Vasilyev, AV, Vasilyev, VB: Periodic Riemann problem and discrete convolution equations. Differ. Equ. 51(5), 652-660 (2015)

12. Eskin, G: Boundary Value Problems for Elliptic Pseudodifferential Equations. Am. Math. Soc., Providence (1981)

13. Gakhov, FD: Boundary Value Problems. Dover, New York (1981)

14. Muskhelishvili, NI: Singular Integral Equations. North-Holland, Amsterdam (1976)

15. Gokhberg, I, Krupnik, N: Introduction to the Theory of One-Dimensional Singular Integral Equations. Birkhäuser, Basel (2010)

\section{Submit your manuscript to a SpringerOpen ${ }^{\ominus}$ journal and benefit from:}

- Convenient online submission

Rigorous peer review

- Immediate publication on acceptance

- Open access: articles freely available online

- High visibility within the field

- Retaining the copyright to your article 